BMJ

Open

Gastroenterology

\section{Bowel ulceration following tocilizumab administration in a COVID-19 patient}

To cite: Bruce-Hickman D, Sajeed SM, Pang YH, et al. Bowel ulceration following tocilizumab administration in a COVID-19 patient. BMJ Open Gastro 2020;7:e000484. doi:10.1136/ bmjgast-2020-000484

Received 6 July 2020 Revised 28 July 2020 Accepted 30 July 2020
Check for updates

(c) Author(s) (or their employer(s)) 2020. Re-use permitted under CC BY-NC. No commercial re-use. See rights and permissions. Published by BMJ.

${ }^{1}$ Intensive Care Medicine, $\mathrm{Ng}$ Teng Fong General Hospital, National University Health System, Singapore 2Department of Pathology, National University Hospital, Singapore

${ }^{3}$ Department of General Surgery, Ng Teng Fong General Hospital, National University Health System, Singapore ${ }^{4}$ Gastroenterology and Hepatology, Ng Teng Fong General Hospital, National University Health System, Singapore

Correspondence to Dr Damian Bruce-Hickman; damian_andrew_david_brucehickman@nuhs.edu.sg

\section{ABSTRACT}

Tocilizumab, a monoclonal antibody against interleukin-6, has been used to treat cytokine release syndrome (CRS) in a subset of patients with severe COVID-19 disease. Acute ulcerative bowel disease has been only rarely documented in patients treated for rheumatological conditions. The gastrointestinal side effects seen when used in the context of COVID-19 are unknown. We present a case of COVID-19 CRS in which acute terminal ileum and perforated caecal ulceration evolved after tocilizumab exposure. We raise awareness of a possible causal relationship between even a single dose of tocilizumab and gut ulceration in patients with COVID-19. Any such drug enteropathy relationship requires watchful monitoring during upcoming trials of tocilizumab in patients with COVID-19.

\section{CASE PRESENTATION}

A 43-year-old man presented to our hospital with a week's history of cough, high fever and anosmia. A nasopharyngeal PCR swab taken on arrival to hospital was positive for SARS-CoV-2 (MiRXES Fortitude 2.1 COVID-19 RT-PCR test). His medical history included gout, hypertension and chronic kidney disease (CKD-3, eGFR 55). Previously, he had suffered from unprovoked lower limb venous thromboembolism (VTE) and was found to be lupus anticoagulant (LAC) positive on two separate occasions in 2017 but had declined anticoagulation (B2-glycoprotein IgG and anti-cardiolipin IgM negative, homocysteine, anti-thrombin III and protein C levels normal).

Our patient was admitted directly to intensive care and had a stormy disease course. On day 1 of hospitalisation, he required intubation for respiratory failure, commencement on dual vasopressors for refractory shock (norepinephrine $\max 0.28 \mathrm{mcg} / \mathrm{kg} / \mathrm{min}$ on day 2, stopped by day 9 of hospitalisation; vasopressin $\max 0.03$ units/min on day 2 , stopped on day 3 of hospitalisation) and instigation of continuous renal replacement therapy. He developed COVID-19 acute respiratory distress syndrome (H-type CARDS) requiring prone-positioning and paralysis.
Progression towards refractory shock, rapidly deteriorating CARDS and high-grade fever prompted review by our institute's multidisciplinary COVID-19 team for possible cytokine release syndrome (CRS). Supportive investigations included an interleukin-6 (IL-6) level of $>1000 \mathrm{pg} / \mathrm{mL}$ (reference range 0.0-3.4 $\mathrm{pg} / \mathrm{mL}$ ), D-dimer $8.56 \mathrm{mcg} / \mathrm{mL}$, ferritin $8109 \mathrm{ng} / \mathrm{mL}(21.8-275.0 \mathrm{ng} / \mathrm{mL})$, Lactate dehydrogenase $2111 \mathrm{U} / \mathrm{L}$ (125-220 U/L) and Aspartate transaminase $1896 \mathrm{U} / \mathrm{L}$ (10-34 $\mathrm{U} / \mathrm{L})$. His overall H-score was 146. Tocilizumab, a monoclonal antibody against IL-6, was administered on day 2 of hospitalisation ( $800 \mathrm{mg}$ via intravenous route) in the face of rapidly deteriorating clinical and biochemical picture, with use of supportive clinicolaboratory scoring. Further doses were withheld due to improvement in clinical status. He received hydrocortisone between days 2 and 5 for shock but received no other targeted COVID-19 intervention (antivirals or other therapies).

Our patient had antecedent LAC positivity with prior unprovoked VTE, and a heparin infusion was started on day 2 of hospitalisation and continued until day 17 when overt lower gastrointestinal (GI) bleeding occurred. Two days prior to overt GI bleeding, high-volume diarrhoea had necessitated rectal tube insertion. On day 17, CT mesenteric angiogram (CTMA) showed arterial blush in the caecum and thickening of the terminal ileum. Embolisation of the culprit branch was performed. Bleeding continued on day 18 of admission; repeated CTMA demonstrated new areas of active bleeding in the ascending colon. A second embolisation was performed of a separate culprit vessel. Despite these two procedures, our patient continued to bleed, requiring significant amounts of blood transfusion. On day 20 of admission, colonoscopy was performed with attempted endostasis. Endoscopy revealed erythematous friable ileal mucosa and luminal bleeding up to at least $15 \mathrm{~cm}$ proximal to the ileocecal valve. 


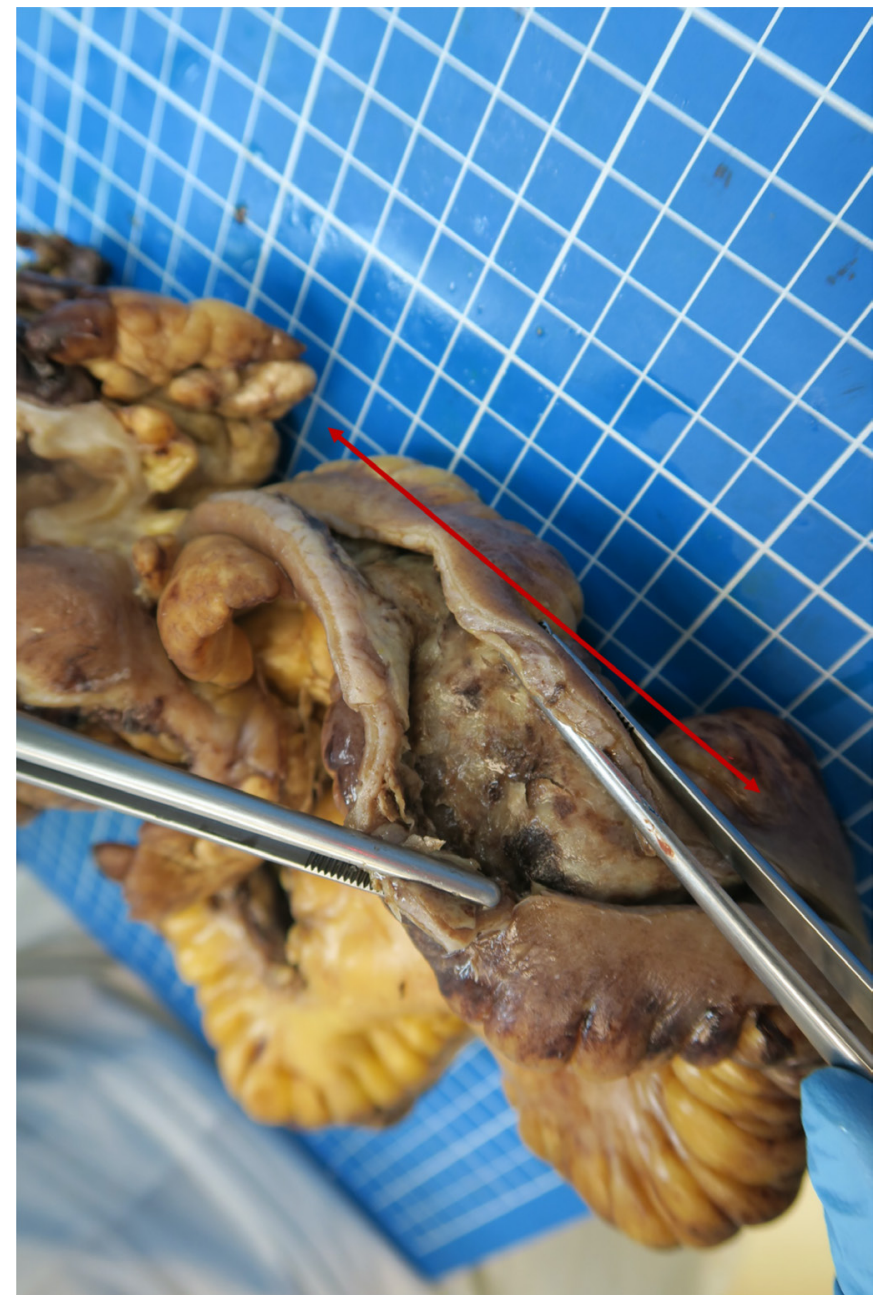

Figure 1 Distal terminal ileum ulceration involving a $30 \mathrm{~cm}$ segment (red arrow).

There was colitis of the caecum and ascending colon $(<50 \%$ of segment) with large ulcerations $(<10 \%$ of segment). Due to failure of endostasis, a right-sided hemicolectomy and segmental resection of the terminal ileum was performed. Gross examination shows extensive ulceration of the distal $30 \mathrm{~cm}$ segment of the terminal ileum associated with thickened dusky wall, fat encroachment and mesenteric stranding (figure 1). The caecum showed three deep linear circumferential ulcers with associated bowel wall perforation. Microscopic examination revealed extensive ulceration of the terminal ileum and deep fissuring ulceration of the caecum associated with perforation. The mucosa at the ulcer edges showed irregular, branching and disordered crypts, compatible with regenerative changes. Apart from foreign material from prior embolisation attempts, there were no thrombi within the large vessels. Smaller vessels around ulcerative sites contained scattered fibrin microthrombi but the overall burden was low, and no ischaemic changes were observed. No features of severe ischaemic colitis, transmural lymphoid aggregates, viral inclusions, granulomas, parasites or vasculitic changes were found (figure 2).
Drug chart review did not identify other culprit drugs (vasopressors had been stopped by day 9 of hospitalisation, hydrocortisone prescribed for 3 days during shocked episode, no non-steroidal anti-inflammatory drugs prescribed). Superimposed infective cause for the gut inflammation observed was excluded. HIV screening was negative, Clostridium difficile testing of stool samples was negative. Tissue bacterial culture yielded light growth of multidrug-resistant Pseudomonas, which had also grown from the respiratory tract and was viewed as a colonising organism. Fungal cultures grew light growth of Candida glabrata, which was also considered non-pathogenic. Acid-fast smears and molecular testing for tuberculosis in excised tissue were negative, as were PCR tests varicellazoster virus. Serology for cytomegalovirus IgM negative, blood PCR for cytomegalovirus and histological analysis for viral inclusions were all negative. Tissue PCR for SARS-CoV-2 from the excised specimen was not possible at our local laboratory. A stool PCR for SARS-CoV-2 was sent prior to laparotomy was negative. After ileostomy formation, a swab taken per stoma was also negative for SARS-CoV-2 PCR.

\section{DISCUSSION}

A subset of patients with severe COVID-19 experience CRS characterised by sustained lymphopenia and elevated cytokines. ${ }^{1}$ This hyperinflammation is associated with increased mortality. ${ }^{2}$ IL-6 may perpetuate CRS and observational data of 21 patients treated with tocilizumab suggested that IL-6 blockade may improve mortality in severe COVID-19 infection. ${ }^{34}$ Expert opinion supports screening patients using laboratory values and clinicolaboratory composite scoring (such as H-scoring) to identify patients at risk of hyperinflammation and potentially
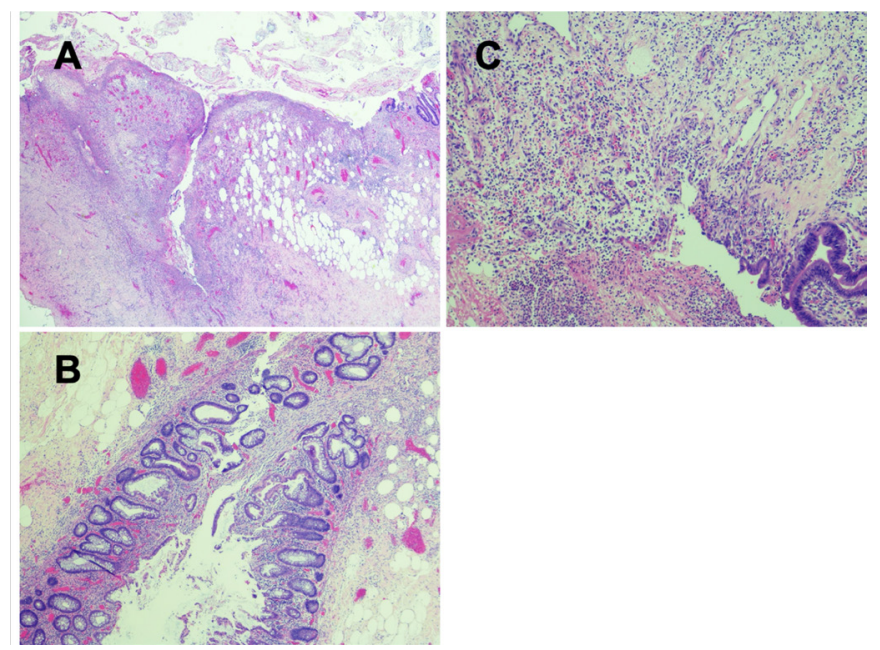

Figure 2 Histological analysis of excised specimen. (A) H\&E stain ( $\times 40$ magnification): Deep fissuring ulceration in caecum. (B) H\&E stain ( $\times 40$ magnification): Irregular branching crypts of ulcer edge tissue. (C) H\&E stain ( $\times 100$ magnification): Ulcer base tissue. No transmural, lymphoid aggregates, basal lymphoplasmacytosis, granulomas, viral inclusions or parasites seen. 
select patients for targeted biological therapy, ${ }^{5}$ although concerns exist over the sensitivity of H-scoring in COVID$19 .^{6}$ The use of tocilizumab in COVID-19 is cautiously supported by the Infectious Diseases Society of America guidelines in the context of clinical trials. ${ }^{7}$ At time of writing, there were 19 studies worldwide actively recruiting for use of tocilizumab in COVID-19 (clinicaltrials.gov).

\section{TOCILIZUMAB AND RISK OF ACUTE GUT INJURY}

Tocilizumab is associated with lower GI perforation (predominantly sigmoid and colonic) and perforated diverticular disease during treatment for rheumatoid arthritis. ${ }^{8}$ As such, these conditions are frequent exclusion criteria in tocilizumab-COVID trials. Acute ulcerative bowel disease is rarely described. We found the report of three cases where tocilizumab appears to have contributed to severe terminal ileum-centred disease. Iwasa and colleagues described a 57-year-old woman with rheumatoid arthritis who presented with haematochezia after 3 months of tocilizumab therapy. ${ }^{9}$ Endoscopic examination showed multiple ulcers in the small and large intestines. Tocilizumab was restarted after a 1-month drug holiday, resulting in recurrence of punched out ulceration in the terminal ileum and deep ulcers in the caecum through to the descending colon. In the second case, a 68-year-old man who had been receiving tocilizumab for 1 year for inflammatory arthritis was found to have pneumatosis intestinalis on a routine chest radiograph. ${ }^{10}$ Withdrawal of tocilizumab led to resolution of the pneumatosis. A follow-up magnetic resonance study of the bowel showed severe stricturing of the terminal ileum, perhaps indicating the site of most intense inflammation. Finally, a 15-year-old boy treated for systemic juvenile arthritis developed abdominal pain and diarrhoea 2 days after his first tocilizumab exposure. ${ }^{11}$ Endoscopy demonstrated minor inflammation in the distal ileum. He received a second dose of tocilizumab and represented 8 days after administration critically unwell secondary to multiple perforations of the terminal ileum. Collectively, these cases provide warning of an ulcerative disease process centred on the terminal ileum which shows temporal relation and possibly a dose-related effect of tocilizumab enteropathy.

\section{THE ROLE OF IL-6 IN GUT HOMEOSTASIS}

IL-6 has protective and reparative roles in acute bowel injury. ${ }^{12-15}$ In mice, injury to the intestine results in IL-6 production by intraepithelial lymphocytes ${ }^{12}$; blockade of IL-6 impairs wound healing by preventing enterocyte proliferation. Translational research demonstrates similar effects in human tissue. In patients undergoing emergency surgery, IL-6 localised to regional traumatic gut perforation but was absent at sites distant to injury. ${ }^{12}$ IL-6 ameliorates gut injury following ischaemia-reperfusion in mice and defends gut barrier integrity. ${ }^{13}$ This is an important consideration for the intensivist managing patients with COVID-19, since tocilizumab has been associated with septic shock secondary to presumed gut translocation of microorganisms. ${ }^{16}$ Patients with COVID-19 requiring intensive care may already risk impaired gut integrity due to systemic hypoperfusion and vasopressor use. ${ }^{17}$ Tocilizumab may further impair gut barrier health in these patients. Taken together, there is evidence experimentally that IL-6 plays an important role in preventing enterocyte cell death, while translational research shows IL-6 is specifically induced at sites of injury in human gut tissue.

\section{SARS-COV-2-RELATED GUT INJURY}

GI symptoms can be elicited in up to $15 \%$ of patients with COVID-19. ${ }^{18}$ SARS-CoV-2 may be detectable in stool in more than $50 \%$ of patients. ${ }^{19}$ There is biological rationale for possible COVID-19 tropism to the ileum. SARS-CoV-2 requires presence of both the cell receptor ACE2 and the transmembrane protease serine 2 (TMPRSS2) for access into cells ${ }^{20}$; both are coexpressed in the oesophagus, lung, ileum and colon. ${ }^{19}$ ACE2 is highly expressed in distal enterocytes ${ }^{20}$ and ACE2 expression in the terminal ileum is almost 10 -fold higher than that in the rectal colon. ${ }^{21}$ In vivo, SARS-CoV-2 is enteropathic. Human intestinal organoids infected with SARS-CoV-2 demonstrate cellular stress and even frank disintegration under high infection burden. ${ }^{20}$ There is paucity of endoscopic studies in patients with COVID-19. Lin and colleagues described erosions and ulcers in the oesophagus of one out of six patients with COVID-19 undergoing endoscopy for GI complaints. ${ }^{22}$ SARS-CoV-2 RNA was detected in biopsies from the oesophagus, stomach, duodenum and rectum in one out of the six patients examined (proctoscopy but not colonoscopy was performed). In a separate cohort of 73 patients in China, a single patient underwent endoscopy for upper GI bleeding. ${ }^{23}$ Although ulcerations were found in the oesophagus, staining for viral nucleocapsid protein was limited to glandular tissues in the stomach, duodenum and rectum and was undetectable in the oesophageal (squamous) epithelium. Autopsy publications reporting GI findings are scant. Stenosis and dilatation of the small bowel has been described in a single patient postmortem; however, it is unclear if these findings can be attributed to COVID-19 disease. ${ }^{24}$ Varga $e t$ al described two cases of mesenteric ischaemia in patients with circulatory failure. Histology from the small bowels of these patients demonstrated diffuse endothelial inflammation and cell apoptosis. ${ }^{25}$ Viral inclusions were demonstrable in the kidney of a third patient without bowel ischaemia but were not found in the gut endothelium of the first two cases.

Evidence for direct erosive SARS-CoV-2 enteropathy sufficient to cause clinically apparent disease remains absent. To our knowledge, despite biological plausibility, there is no confirmation of SARS-CoV-2 presence in the terminal ileum nor histological evidence of SARS-CoV-2 enteropathy in the small bowel to date. 
The surgical team at Massachusetts General Hospital have published both case study and case series of COVIDrelated gut complications. ${ }^{26}{ }^{27}$ Out of 141 patients, 58 had ileus, 2 pseudo-obstruction, 1 had extensive hepatic necrosis, and 4 had bowel ischaemia requiring resection. ${ }^{27}$ One of the four patients had pulmonary embolism, extensive bowel necrosis and terminal ileum perforation on exploratory laparotomy. ${ }^{27}$ The antimesenteric pattern of necrosis observed was attributed to microthrombus and hypercoagulability. Patients with COVID-19 have elevated fibrinogen and D-dimer levels, which correlate with mortality, and point-of-care thromboelastometry demonstrates hypercoagulopathic profiles. ${ }^{28}$ Microthrombi are frequently observed in the lungs postmortem ${ }^{29}$; however, viral-PCR testing and histopathological studies of the colon and ileum have not been reported in these cohorts to date. ${ }^{30}$ Endotheliitis has been documented in excised small bowel in patients suffering COVID-19 and mesenteric ischaemia. It seems unlikely that endotheliitis would be responsible for widespread bowel necrosis however, and both patients described had superimposed circulatory dysfunction which may have contributed to the ischaemia observed. ${ }^{25}$ Our patient had antecedent unprovoked deep vein thrombosis and LAC positivity which may have further increased his prothrombotic risk; however, no evidence of significant microthrombi nor ischaemia was seen on histological analysis.

\section{CONCLUSIONS AND LEARNING POINTS}

We describe, to our knowledge, the first case of severe ulcerative disease in the context of tocilizumab therapy in a COVID-19-positive patient. This case study joins a growing body of reports identifying acute ulceration focused on the terminal ileum and temporally related to tocilizumab exposure.

Factors other than direct drug enteropathic effect should be considered. SARS-CoV-2 is enterotropic, yet whether COVID-19 has specific tropism for the ileocecal region in infected humans is unproven. We do not have evidence of SARS-CoV-2 gut infection in our own patient since stool and stomal PCR tests were negative, and we cannot find supporting literature for a clinically important enteropathy effect in infected humans. IL-6 is important in gut homeostasis. Although the temporal relation of tocilizumab administration and ulceration in our patient remains highly suspect, we cannot exclude that tocilizumab is acting as a permissive rather than culprit agent and may simply block adequate healing mechanisms in a gut injured from alternative disease processes (viral enteropathy, microthrombus disease, global mesenteric hypoperfusion from critical illness state and vasopressor use).

A possible causal relationship between even a single dose of tocilizumab and gut ulceration in COVID-19 populations needs to be confirmed by larger observational studies and carefully monitored for in upcoming trials of tocilizumab in patients with COVID-19 CRS.
The incidence of such an association in patients with COVID-19 is unknown and requires further investigation.

Contributors DB-H wrote the first draft of the manuscript. YHP provided histological examination of the bowel and was a major contributor to the histological discussions of the text. SMS, CSS, WC, MGK, YHP and DBH all edited further drafts prior to finalisation as well as reading and approving the final submission.

Funding The authors have not declared a specific grant for this research from any funding agency in the public, commercial or not-for-profit sectors.

Competing interests None declared.

Patient consent for publication Obtained.

Provenance and peer review Not commissioned; externally peer reviewed. Data availability statement № data are available.

Open access This is an open access article distributed in accordance with the Creative Commons Attribution Non Commercial (CC BY-NC 4.0) license, which permits others to distribute, remix, adapt, build upon this work non-commercially, and license their derivative works on different terms, provided the original work is properly cited, appropriate credit is given, any changes made indicated, and the use is non-commercial. See: http://creativecommons.org/licenses/by-nc/4.0/.

ORCID iD

Damian Bruce-Hickman http://orcid.org/0000-0003-1698-5775

\section{REFERENCES}

1 Zhang C, Wu Z, Li J-W, et al. Cytokine release syndrome in severe COVID-19: interleukin- 6 receptor antagonist tocilizumab may be the key to reduce mortality. Int J Antimicrob Agents 2020;55:105954.

2 Ruan Q, Yang K, Wang W, et al. Clinical predictors of mortality due to COVID-19 based on an analysis of data of 150 patients from Wuhan, China. Intensive Care Med 2020;46:846-8.

$3 \mathrm{Xu}$ X, Han M, Li T, et al. Effective treatment of severe COVID-19 patients with tocilizumab. Proc Natl Acad Sci U S A 2020;117:10970-5.

4 Cellina M, Orsi M, Bombaci F, et al. Favorable changes of CT findings in a patient with COVID-19 pneumonia after treatment with tocilizumab. Diagn Interv Imaging 2020.

5 Mehta P, McAuley DF, Brown M, et al. COVID-19: consider cytokine storm syndromes and immunosuppression. Lancet 2020;395:1033-4.

6 Leverenz DL, Tarrant TK. Is the HScore useful in COVID-19? Lancet 2020;395:e83.

7 Bhimraj A, Morgan RL, Shumaker AH, et al. Infectious diseases Society of America guidelines on the treatment and management of patients with COVID-19. Clin Infect Dis 2020:ciaa478.

8 Strangfeld A, Richter A, Siegmund B, et al. Risk for lower intestinal perforations in patients with rheumatoid arthritis treated with tocilizumab in comparison to treatment with other biologic or conventional synthetic DMARDs. Ann Rheum Dis 2017;76:504-10.

9 Iwasa T, Nakamura K, Ogino H, et al. Multiple ulcers in the small and large intestines occurred during tocilizumab therapy for rheumatoid arthritis. Endoscopy 2011;43:70-2.

10 Jacobs B, Jawad A, Fattah Z. Pneumatosis intestinalis and intestinal perforation in a patient receiving tocilizumab. Arch Rheumatol 2018;33:372-5.

11 Pfeil J, Grulich-Henn J, Wenning D, et al. Multiple upper gastrointestinal perforations in a 15-year-old patient treated with tocilizumab. Rheumatology 2014;53:1713-4.

12 Kuhn KA, Manieri NA, Liu T-C, et al. IL-6 stimulates intestinal epithelial proliferation and repair after injury. PLOS One 2014;9:e114195-18.

13 Jin X, Zimmers TA, Zhang Z, et al. Interleukin-6 is an important in vivo inhibitor of intestinal epithelial cell death in mice. Gut 2010;59:186-96.

14 Rollwagen FM, Madhavan S, Singh A, et al. II-6 protects enterocytes from hypoxia-induced apoptosis by induction of bcl-2 mRNA and reduction of fas mRNA. Biochem Biophys Res Commun 2006;347:1094-8.

15 Tebbutt NC, Giraud AS, Inglese M, et al. Reciprocal regulation of gastrointestinal homeostasis by SHP2 and STAT-mediated trefoil gene activation in gp130 mutant mice. Nat Med 2002;8:1089-97.

16 Xu M, Liu C, Guo L, et al. Severe sepsis caused by serious gastrointestinal infection in SJIA patients treated with IL-6 receptor antagonist: a case report. BMC Pediatr 2020;20. 
17 Vikse J, Henry BM. Tocilizumab in COVID-19: beware the risk of intestinal perforation. Int J Antimicrob Agents 2020;56:106009.

18 Mao R, Qiu Y, He J-S, et al. Manifestations and prognosis of gastrointestinal and liver involvement in patients with COVID-19: a systematic review and meta-analysis. Lancet Gastroenterol Hepatol 2020;5:667-78.

$19 \mathrm{Ng} \mathrm{SC}$, Tilg H. COVID-19 and the gastrointestinal tract: more than meets the eye. Gut 2020;69:973-4.

20 Lamers MM, Beumer J, van der Vaart J, et al. SARS-CoV-2 productively infects human gut enterocytes. Science 2020;369:50-4

21 Wang H-G, Xie R, Ma T-H, et al. Excessive anxiety in IBD patients is unnecessary for COVID-19. Clin Res Hepatol Gastroenterol 2020. doi:10.1016/j.clinre.2020.03.001. [Epub ahead of print: 11 May 2020].

22 Lin L, Jiang X, Zhang Z, et al. Gastrointestinal symptoms of 95 cases with SARS-CoV-2 infection. Gut 2020;69:997-1001.

23 Xiao $\mathrm{F}$, Tang $\mathrm{M}$, Zheng $\mathrm{X}$, et al. Evidence for gastrointestinal infection of SARS-CoV-2. Gastroenterology 2020;158:1831-3.
24 Liu Q, Wang RS, Qu GQ, et al. Gross examination report of a COVID-19 death autopsy. Fa Yi Xue Za Zhi 2020;36:21-3.

25 Varga Z, Flammer AJ, Steiger P, et al. Endothelial cell infection and endotheliitis in COVID-19. The Lancet 2020;395:1417-8

26 Kaafarani HMA, EI MM, Hwabejire JO, et al. Gastrointestinal complications in critically III patients with COVID-19. Ann Surg 2020.

27 Gartland RM, Velmahos GC. Bowel necrosis in the setting of COVID-19. J Gastrointest Surg 2020:1-2.

28 Spiezia L, Boscolo A, Poletto F, et al. COVID-19-Related severe hypercoagulability in patients admitted to intensive care unit for acute respiratory failure. Thromb Haemost 2020;120:998-1000.

29 McGonagle D, O'Donnell JS, Sharif K, et al. Immune mechanisms of pulmonary intravascular coagulopathy in COVID-19 pneumonia Lancet Rheumatol 2020:1-9.

30 Mao R, Liang J, Shen J, et al. Implications of COVID-19 for patients with pre-existing digestive diseases. Lancet Gastroenterol Hepatol 2020;5:425-7. 